\title{
High prevalence of Neisseria gonorrhoeae strains with reduced susceptibility to fluoroquinolones in
} Japan

\author{
Masatoshi Tanaka, Joichi Kumazawa, Testuro Matsumoto, Intetsu Kobayashi
}

\begin{abstract}
Objective-To study the antibiotic susceptibility of Neisseria gonorrhoeae strains isolated in Japan and, in particular, to examine the possibility of emerging fluoroquinolone resistance.

Materials and methods-Sixty-nine strains of Neisseria gonorrhoeae isolated in 1992 were tested for susceptibility to 15 antibiotics including fluoroquinolones and were seroclassified. Twenty-seven strains isolated from 1981 to 1984 were also evaluated as controls.

Results-The MIC90 values of norfloxacin, ofloxacin, and ciprofloxacin against the isolates from 1992 were $2 \cdot 0$, $1 \cdot 0$, and $0.5 \mu \mathrm{g} / \mathrm{ml}$, respectively. The MIC90 values of norfloxacin, ofloxacin, and ciprofloxacin against the isolates from 1981-84 (controls) were $0 \cdot 25,0 \cdot 125$, and $0.063 \mu \mathrm{g} / \mathrm{ml}$, respectively. These results indicate that the MIC90 values of norfloxacin, ofloxacin, and ciprofloxacin against the strains from 1992 were 8-fold higher than those against the strains from 1981-84. However, there were no significant differences in susceptibility to $\beta$-lactams, tetracyclines, macrolides, and spectinomycin between the isolates from 1992 and those from 1981-84. The majority of the isolates belonged to the WIIWIII serogroup. There was no relationship between fluoroquinolone resistance and serogroup.
\end{abstract}

Conclusions-Fluoroquinolones have been used frequently as first-line therapy and have provided excellent clinical efficacy for gonococcal infections for the last several years in Japan. However, our data indicate that a rapid decrease in the susceptibility of Neisseria gonorrhoeae to fluoroquinolones is occurring in our country.

(Genitourin Med 1994;70:90-93)

University, 3-1-1

Maidashi, Higashi-ku,

Fukuoka 812, Japan

M Tanaka

J Kumazawa

T Matsumoto

Division of

Chemotherapy,

Mitsubishi Yuka BCL

3-30-1 Shimura,

Itabashi-ku, Tokyo,

174, Japan

I Kobayashi

Address correspondence to: Dr M Tanaka

Accepted for publication

1 December 1993

Fluoroquinolones such as ciprofloxacin, norfloxacin, and ofloxacin have excellent in vitro antimicrobial activity against Neisseria gonorrhoeae, including penicillin- and tetracyclineresistant strains, and are highly effective as oral single-dose treatment for gonococcal infections. ${ }^{1-3}$ Treatment of gonorrhoea with ciprofloxacin is recommended by the World Health Organization (WHO). ${ }^{4}$ In Japan, fluo- first-line therapy for gonorrhoea for several years.

However, reduced sensitivity to ciprofloxacin $^{56}$ and treatment failures with this agent have recently been reported. ${ }^{5}$ Development of fluoroquinolone resistance in Neisseria gonorrhoeae may be a new worldwide problem complicating the treatment of gonococcal infections.

The present study was performed to investigate the antibiotic susceptibility of Neisseria gonorrhoeae isolates in Japan, and particularly to examine the possibility of emerging fluoroquinolone resistance.

\section{Materials and methods \\ Bacterial strains}

A total of 82 strains of Neisseria gonorrhoeae obtained from men with urethritis attending 15 sexually transmitted disease clinics between February and July 1992 were included in this study. Clinical and epidemiologic information was recorded on the patient's name, age, sexual preference, locality of contact, presence and duration of symptoms, prior antimicrobial exposure, and treatment throughout the study period. Out of the 82 strains, 13 were excluded, that were posttreatment isolates or repeat isolates from the same patient. The remaining 69 strains were tested. Specimens from each patient were inoculated directly onto Thayer-Martin agar (BBL), transported to the laboratory and incubated for 24-48 hours in a 5\% $\mathrm{CO}_{2}$ atmosphere. Neisseria gonorrhoeae was identified as Gram-negative diplococci and by oxidase reaction and sugar utilisation patterns. The isolates were stored at $-80^{\circ} \mathrm{C}$ in GDO medium (Nissui) containing gelatin with $10 \%$ skim milk, $2 \%$ activated charcoal, and $17 \%$ glucose until tested.

For comparative purposes, 27 Neisseria gonorrhoeae strains (14 $\beta$-lactamase-producing and 13 non- $\beta$-lactamase-producing) that had been isolated between 1981 and 1984 and stored at $-80^{\circ} \mathrm{C}$ in our laboratory were also evaluated as controls.

\section{Introduction} roquinolones have been frequently used as

\section{Antibiotic susceptibility testing}

The minimum inhibitory concentration (MIC) for all isolates was determined using an agar dilution technique with a GC agar base (Difco) containing $1 \%$ Iso VitaleX (BBL) and 2-fold dilutions of antibiotic. Plates were inoculated with $5 \mu 1$ of $10^{6}$ colonyforming units $(\mathrm{cfu}) / \mathrm{ml}$ of each isolate using a multipoint inoculator. World Health 
Table 1 MICs of antimicrobial agents against Neisseria gonorrhoeae isolated in fapan

\begin{tabular}{|c|c|c|c|c|c|c|}
\hline \multirow[b]{3}{*}{ Antibiotics } & \multicolumn{6}{|c|}{$M I C(\mu g / m l)$} \\
\hline & \multicolumn{3}{|c|}{$1992(n=69)$} & \multicolumn{3}{|c|}{$1981-84(n=27)$} \\
\hline & $50 \%$ & $90 \%$ & Range & $50 \%$ & $90 \%$ & Range \\
\hline $\begin{array}{l}\text { Norfloxacin } \\
\text { Ofloxacin } \\
\text { Ciprofloxacin } \\
\text { Erythromycin } \\
\text { Azithromycin } \\
\text { Tetracycline } \\
\text { Doxycycline } \\
\text { Spectinomycin } \\
\text { Cefotaxime } \\
\text { Ceftriaxone } \\
\text { Cefixime } \\
\text { Aztreonam } \\
\text { Imipenem } \\
\text { Penicillin G* } \\
\text { Piperacillin* }\end{array}$ & $\begin{array}{l}0.5 \\
0.5 \\
0.063 \\
0.5 \\
0.125 \\
1.0 \\
1.0 \\
16.0 \\
0.031 \\
0.016 \\
0.016 \\
0.125 \\
0.063 \\
0.25 \\
0.063\end{array}$ & $\begin{array}{l}2 \cdot 0 \\
1 \cdot 0 \\
0.5 \\
2 \cdot 0 \\
0 \cdot 25 \\
2 \cdot 0 \\
1 \cdot 0 \\
16 \cdot 0 \\
0 \cdot 125 \\
0.063 \\
0.031 \\
0.5 \\
0.125 \\
1.0 \\
0.125\end{array}$ & $\begin{aligned} 0.004-8.0 \\
0.002-2.0 \\
\leqslant 0.001-0.5 \\
0.063-2.0 \\
0.016-0.5 \\
0.125-4.0 \\
0.125-4.0 \\
4.0-32 \cdot 0 \\
0.004-0.25 \\
0.004-0.063 \\
0.002-0.063 \\
0.031-0.5 \\
0.008-0.5 \\
0.016-2.0 \\
\leqslant 0.008-0.25\end{aligned}$ & $\begin{array}{l}0.063 \\
0.063 \\
0.008 \\
0.5 \\
0.125 \\
1.0 \\
1.0 \\
16.0 \\
0.031 \\
0.016 \\
0.016 \\
0.125 \\
0.063 \\
1.0 \\
0.125\end{array}$ & $\begin{array}{l}0.25 \\
0.125 \\
0.063 \\
1.0 \\
0.25 \\
4.0 \\
2.0 \\
16.0 \\
0.125 \\
0.063 \\
0.063 \\
0.25 \\
0.125 \\
2.0 \\
0.25\end{array}$ & $\begin{array}{l}0.004-2.0 \\
0.004-1.0 \\
\leqslant 0.001-0.25 \\
0.063-2.0 \\
0.031-0.5 \\
0.25-4.0 \\
0.125-4.0 \\
8.0-32.0 \\
0.004-0.25 \\
0.002-0.063 \\
0.004-0.063 \\
0.031-0.5 \\
0.008-0.5 \\
0.063-2.0 \\
\leqslant 0.008-0.5\end{array}$ \\
\hline
\end{tabular}

${ }^{\star}$ non-PPNG strains only: $1992 ; n=67,1981-84 ; n=13$

Organization Neisseria gonorrhoeae reference strains A-E (kindly supplied by Dr. J. W. Tapsall, The Prince of Wales Hospital, Australia) were included as quality standards. The plates were incubated for 24 hours at $35^{\circ} \mathrm{C}$ in a $5 \% \mathrm{CO}_{2}$ atmosphere. MICs were read as the lowest concentration of antibiotic that inhibited growth.

The tested antibiotics were norfloxacin, ofloxacin, ciprofloxacin, erythromycin, azithromycin, tetracycline, doxycycline, spectinomycin, cefotaxime, ceftriaxone, cefixime, aztreonam, imipenem, penicillin $G$, and piperacillin. All of the antibiotics were obtained as powders of stated potency from their manufacturers. $\beta$-lactamase production was tested by an acidometric assay (Oxoid).

Serological classification

Classification into serogroups (WI and WII/WIII) was performed by coagglutination using the Phadebact Monoclonal GC Test ${ }^{\mathrm{TM}}$ (Pharmacia).

\section{Statistical analysis}

The proportions of isolates from 1992 with reduced sensitivity to norfloxacin, ofloxacin, and ciprofloxacin were compared with those of isolates from 1981-84 using the $\chi^{2}$ test. There are no established categories that define reduced sensitivity to these antibiotics.

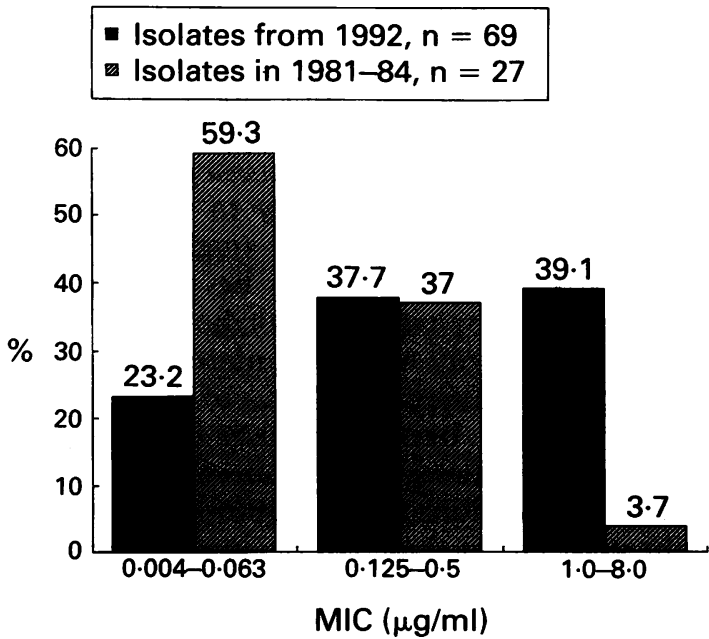

Figure 1 Distribution of norfloxacin susceptibility.
Therefore, we chose three categories of sensitivity as follows: $\leqslant 0.063,0.125-0.5$, and $\geqslant 1.0 \mu \mathrm{g} / \mathrm{ml}$ for norfloxacin; $\leqslant 0.016$, $0.031-0.25$, and $\geqslant 0.5 \mu \mathrm{g} / \mathrm{ml}$ for ofloxacin; $\leqslant 0.008, \quad 0.016-0.063$, and $\geqslant 0.125$ for ciprofloxacin. The MICs in the third category indicate reduced sensitivity.

\section{Results}

Antibiotic susceptibility

The MIC50, MIC90, and MIC ranges of the 15 tested antimicrobial agents against the 69 isolates from 1992 and the 27 isolates from $1981-84$ are shown in table 1 . Only $2(2.9 \%)$ of the 69 strains from 1992 were $\beta$-lactamaseproducing Neisseria gonorrhoeae (PPNG).

Therefore, for the studied penicillins the results of the non-PPNG strains were compared. The MIC90 value of norfloxacin against the isolates from 1992 was $2 \cdot 0 \mu \mathrm{g} / \mathrm{ml}$, while that against the isolates from 1981-84 was $0.25 \mu \mathrm{g} / \mathrm{ml}$. Therefore, the MIC90 value of norfloxacin against the isolates from 1992 was 8-fold higher than that against isolates from 1981-84. The MIC90 value of ofloxacin against the strains from $1992(1.0 \mu \mathrm{g} / \mathrm{ml})$ was also 8-fold higher than that against the strains from 1981-84 $(0 \cdot 125 \mu \mathrm{g} / \mathrm{ml})$. Furthermore, the MIC90 value of ciprofloxacin against the strains from $1992(0.5 \mu \mathrm{g} / \mathrm{ml})$ was 8 -fold higher than that against the strains from 1981-84 $(0.063 \mu \mathrm{g} / \mathrm{ml})$.

In general, the MIC90 values of the other tested antibiotics against the isolates from 1992 were equal, 2-fold higher, or 2-fold lower than those against the isolates from 1981-84.

The incidence of strains from 1992 with reduced sensitivity to norfloxacin $(39 \cdot 1 \%)$ was significantly higher than that of strains from 1981-84 (3.7\%; $p<0.001$ fig 1$)$. There was also a significant difference in the incidence of reduced sensitivity to ofloxacin between the strains from $1992(40.6 \%)$ and those from 1981-84 (3.7\%; p < 0.001, fig 2). Furthermore, a significantly higher incidence of reduced sensitivity. to ciprofloxacin was observed in the strains from $1992(47 \cdot 8 \%)$, compared with those from 1981-84 (3.7\%; p $<0.001$, fig 3 ).



Figure 2 Distribution of ofloxacin susceptibility. 
Figure 3 Distribution of ciprofloxacin susceptibility.

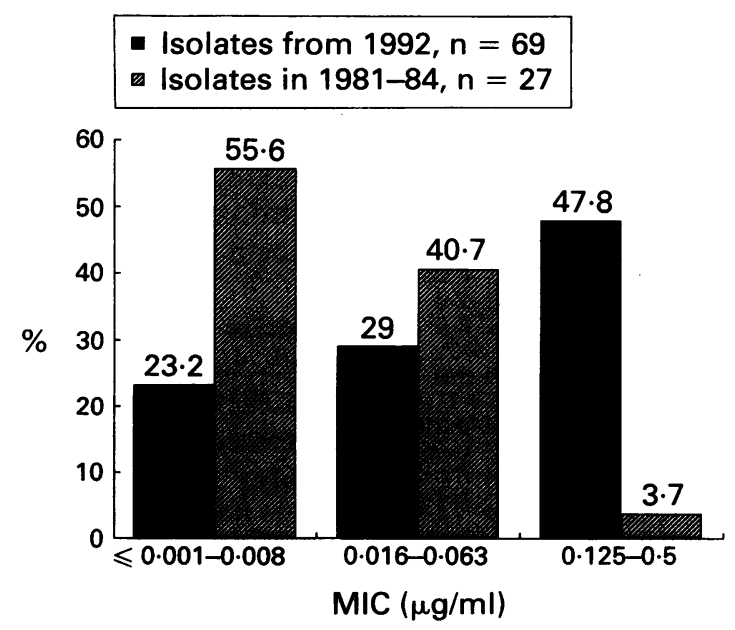

Table 2 Serogroups of Neisseria gonorrhoeae strains isolated in Fapan

\begin{tabular}{llc}
\hline & \multicolumn{2}{l}{ No. of strains (\%) } \\
\cline { 2 - 3 } Serogroups & 1992 & $1981-84$ \\
\hline WI & $2(2 \cdot 9)$ & 0 \\
WII/WIII & $66(95 \cdot 7)$ & $27(100)$ \\
WI and WII/WIII & $1(1 \cdot 4)$ & 0 \\
Total & $69(100)$ & $27(100)$ \\
\hline
\end{tabular}

Serological classification

Of the 69 isolates from 1992, $66(95 \cdot 7 \%)$ belonged to the WII/WIII serogroup, 2 $(2 \cdot 9 \%)$ belonged to the WI serogroup, and 1 $(1.4 \%)$ reacted with both WI and WII/WIII, while all $27(100 \%)$ isolates from 1981-84 belonged to the WII/WIII serogroup (table 2). Most of the isolates were in the WII/WIII serogroup. Therefore, there was no relationship between fluoroquinolone resistance and serogroup.

\section{Discussion}

Gonococcal resistance to antimicrobial agents is an increasing problem in the treatment of gonorrhoea. A high prevalence of plasmidmediated high-level or chromosomally mediated low-level resistance to penicillin or tetracycline has been reported in southeast Asia $^{7-10}$ and in African countries. ${ }^{11-13}$

Oral single-dose treatment with a fluoroquinolone is now indicated as first-line therapy for gonorrhoea in countries with the problem of antibiotic multiresistance because fluoroquinolones have strong antimicrobial activity against Neisseria gonorrhoeae, including strains resistant to penicillin and tetracycline. In addition, oral single-dose treatment is very convenient and cheap. However, the appearance of strains showing reduced sensitivity to ciprofloxacin in vitro ${ }^{56}$ and treatment failure with the antibiotic have recently been reported in the United Kingdom, ${ }^{5}$ although the prevalence of strains resistant to fluoroquinolones is not yet a major problem in that country..$^{14} 15$

Our results demonstrated that there was significantly higher prevalence of reduced sensitivity to norfloxacin, ofloxacin, and ciprofloxacin among isolates from 1992, compared with isolates from 1981-84. The MIC90 values of norfloxacin, ofloxacin, and ciprofloxacin against the strains from 1992 were $2.0,1.0$, and $0.5 \mu \mathrm{g} / \mathrm{ml}$, respectively, while those against the strains from 1981-84 were $0.25,0.125$, and $0.063 \mu \mathrm{g} / \mathrm{ml}$, respect tively. A decrease in susceptibility of gonocog cal isolates to fluoroquinolones has also been observed in the Philippines. Clendennen et al= reported that the MIC90 values of nor floxacin, ofloxacin, and ciprofloxacin against Neisseria gonorrhoeae isolated in 1989 in the Philippines were $2 \cdot 0,0.625$, and $0.25 \mu \mathrm{g} / \mathrm{m}$ respectively. Therefore, the MIC90 values of fluoroquinolones against Neisseria gonorrhoed in the Philippines are similar to those of is $\mathbb{D}$ lates from 1992 in our country. Furthermore in Rwanda ${ }^{13}$ decreasing susceptibility of gono cocci to norfloxacin and ofloxacin has been observed. Although Neisseria gonorrhoed $\vec{g}$ strains with resistance to fluoroquinolones are not yet widespread in the United Kingdom, ${ }^{14} \stackrel{\sqrt[5]{5}}{\sqrt{5}}$ Australia, ${ }^{16}$ New Zealand, ${ }^{17}$ Thailand, ${ }^{8}$ Hon 8 Kong, ${ }^{10}$ Zaire, ${ }^{11}$ and the Gambia, ${ }^{12}$ the increase in fluoroquinolone-resistant Neisserie gonorrhoeae strains may become a major problem in these countries in the future.

The reasons for this outbreak of Neisseria gonorrhoeae strains with reduced sensitivity to fluoroquinolones in Japan are not clear. Onf of the major reasons may be that fluoro quinolones have been increasingly used empirical first-line treatment for gonorrhoe for the last several years in our countrot Another possibility is that a new strain of fluog roquinolone-resistant Neisseria gonorrhoea was introduced into Japan from southeast Asian countries such as the Philippines.

There is little information about the rela tionship between the in vitro MIC of fluoro quinolones against gonococci and clinicat efficacy. Gransden et $a l^{5}$ reported that clinica failure with ciprofloxacin occurred in a patier with an Neisseria gonorrhoeae strain showing an MIC of $0.06 \mu \mathrm{g} / \mathrm{ml}$ treated with a $250-\mathrm{mg} \sin 5$ gle dose of ciprofloxacin. Bogaerts et al reported that failure with norfloxacin occurred more often in patients with strain having MICs $\geqslant 0.06 \mu \mathrm{g} / \mathrm{ml}$ treated with $800^{\circ}$ $\mathrm{mg}$ single doses. In the present study, we measured the in vitro antimicrobial activity of fluoroquinolones against Neisseria gonorrhoeas but did not evaluate the clinical efficacy of the agents. Because of the high prevalence of Neisseria gonorrhoeae isolates with low suscepti bility to fluoroquinolones in Japan, it seem that single-dose treatment with these agen may be problematic as first-line therapy fof gonorrhoea in our country. Therefore, the antimicrobial sensitivity of Neisseria gono rhoeae to fluoroquinolones and the therapeutic efficacy of these antibiotics shoud be close monitored.

Quinolone resistance in gram-negative bacteria involves alterations in DNA gyrase and the permeability of bacteria membranes. ${ }^{18}$ Inactivation of quinolones by enzymatic degradation as a mechanism of bacterial resistance has not been reported, and 
plasmid-mediated resistance to quinolones has not been proved. The research on mechanisms of quinolone resistance in gonococci is very sparse. One study showed that reduced uptake in a ciprofloxacin-resistant strain of Neisseria gonorrhoeae could be a mechanism of gonococcal resistance to this agent. ${ }^{19}$ However, the ciprofloxacin-resistant Neisseria gonorrhoeae tested in that study was only one strain. Further investigations of the mechanisms of quinolone resistance in Neisseria gonorrhoeae are indicated.

There were no significant differences in susceptibility to $\beta$-lactams, tetracyclines, macrolides, and spectinomycin between our isolates from 1992 and those from 1981-84. The incidence of PPNG (2.9\%) in this study was lower than the incidence of $10 \%$ to $24 \%$ previously reported in Japan between 1983 and $1986 .{ }^{20}$ The observed low prevalence of PPNG may be associated with an increase in the use of fluoroquinolones for gonococcal infections in our country.

This study was supported by Pfizer Pharmaceuticals Inc., Japan.

1 Loo PS, Ridgway GL, Oriel JD. Single dose ciprofloxacin for treating gonococcal infections in men. Genitourin Med 1985;61:302-5.

2 Panikabutra K, Lee CT, Ho B, Bamberg P. Single dose oral norfloxacin or intramuscular spectinomycin to treat gonorrhoea (PPNG and non-PPNG infections): analysis of efficacy and patient preference. Genitourin Med 1988; 64:235-40.

3 Black JR, Long JM, Zwickl BE, et al. Multicent er randomized study of single-dose ofloxacin versus amoxicillinprobenecid for treatment of uncomplicated gonococcal infection. Antimicrob Agents Chemother 1989;33:167-70.

4 World Health Organization. STD Treatment Strategies. Geneva. World Health Organization 1989; WHO/ VDT/89.447.
5 Gransden WR, Warren CA, Phillips I, Hodges, Barlow D. Decreased susceptibility of Neisseria gonorrhoeae to ciprofloxacin. Lancet 1990;335:51.

6 Jephcott AE, Turner A. Ciprofloxacin resistance in gonococci. Lancet 1990;335:165.

7 Clendennen III TE, Hames CS, Kees ES, et al. In vitro antibiotic susceptibilities of Neisseria gonorrhoeae isolates in the Philippines. Antimicrob Agents Chemother 1992;36: in the Philip

8 Clendennen TE, Echererria P, Saengeur S, et al. Antibiotic susceptibility survey of Neisseria gonorrhoeae in biotic susceptibility survey of Neisseria gonorrhoeae in

$9 \mathrm{Chu}$ ML, Ho LJ, Lin HC, Wu YC. Epidemiology of penicillin-resistant Neisseria gonorrhoeae isolated in Taiwan, 1960-1990. Clin Infect Dis 1992;14:450-7.

10 Kam KM, Lai CF, Egglestone S, Chan CBB. Patterns of antibiotic susceptibility of gonococci isolated in Hong Kong, 1987-1990. Sex Transm Dis 1992;19:284-7.

11 Van Dyck E, Rossau R, Duhamel M, et al. Antimicrobial susceptibility of Neisseria gonorrhoeae in Zaire: high level plasmid-mediated tetacycline resistance in Central Africa. Genitourin Med 1992;68:111-6.

12 Ison CA, Pepin J, Roope NS, Demba E, Secka O, Easmon CSF. The dominance of a multiresistant strain of Neisseria gonomhoeae among prostitutes and STD peisseria gonorrhoeae among prostitutes and STD patients

13 Bogaerts J, Tello WM, Akingeneye J, Mukantabana V, Van Dyck E, Piot P. Effectiveness of norfloxacin and ofloxacin for treatment of gonorrhoea and decrease of in vitro susceptibility to quinolones over time in Rwanda. Genitourin Med 1993;69:196-200.

14 Gransden WR, Warren C, Phillips I. 4-quinolone-resistant Neisseria gonorrhoeae in the United Kingdom. F Med Microbiol 1991;34:23-7.

15 Ison CA, Branley NS, Kirtland K, Easmon CS. Surveillance of antibiotic resistance in clinical isolated of Neisseria gonorrhoeae. BMF 1991;303:1307.

16 Tapsall JW, Shultz TR, Phillips EA. Characteristics of Neisseria gonorrhoeae isolated in Australia showing decreased sensitivity to quinolone antibiotics. Pathology 1992;24:27-31

17 Brett MSY, Davies HGD, Blockley JR, Heffernan HM. Antibiotic susceptibilities, serotypes and auxotypes of Neisseria gonorrhoeae isolated in New Zealand. Genitourin Med 1992;68:321-4.

18 Wolfson JS, Hooper DC. Bacterial resistance to quinolones: mechanisms and clinical importance. Rev Infect Dis 1989;11(suppl):960-8.

19 Corkill JE, Percival A, Lind M. Reduced uptake of ciprofloxacin in a resistant strain of Neisseria gonorrhoeae ciprofioxacin in a resistant strain of Neisseria gonorrhoeae and transformation of resistance to

20 Nishimura M, Kumamoto Y, Hirose T, Sakai S, Tsukamoto T, Deguchi K. Bacteriologic studies on Neisseria gonorrhoeae isolated in Sapporo, Japan. Sex Transm Dis 1991;18:80-3. 